\title{
Eye Manifestations in Patients with Chronic Renal Failure not on Dialysis: A Report about 60 Cases
}

\author{
Koman CE1*, Kouassi FX'1, Sowagnon TYC ${ }^{1}$, Soumahoro M11, Kra \\ ANS11, Ouonnebo LFA², Kouakou AAK ${ }^{1}$, Dibi CAU1 ${ }^{1}$ and Ndoli ADA ${ }^{1}$ \\ ${ }^{1}$ Department of Ophthalmology, Cocody University Hospital, Côte d'Ivoire \\ ${ }^{2}$ Department of Ophthalmology, Côte d'Ivoire
}

*Corresponding author: Koman CE, Department of Ophthalmology, Cocody University Hospital, BPV 13 Abidjan, Côte d'Ivoire, Email: kellalie@outlook.com

\section{Research Article \\ Volume 3 Issue 5}

Received Date: October 17, 2018

Published Date: November 14, 2018

DOI: $10.23880 /$ oajo-16000166

\section{Abstract}

Objectives: The objective of this study was to describe eye manifestations encountered in patients with chronic renal failure who are not undergoing dialysis to ensure better management.

Materials and methods: This was a cross-sectional and descriptive study, carried out from February 2017 to June 2017. The study was conducted jointly in the Nephrology and Ophthalmology departments of Yopougon University Hospital. It focused on patients with renal failure who were not on dialysis. A total of 60 patients were included with respect to the inclusion criteria of the study.

Results: Patients' personal history was dominated by high blood pressure (88\%) and diabetes (27\%). Loss of visual acuity and pruritus were the most expressed symptoms at rates of $66.66 \%$ and $26 \%$, respectively. Biomicroscopic examination of the anterior segment revealed unilateral cataract in $26.67 \%$ of cases. At the posterior pole, optic neuropathies were predominant (23.33\%). Macular edema was 10\%. Arterial narrowing (16.66\%) was the most observed lesion in peripheral chorioretin. Hypertensive Retinopathy (HR) and Large Optic Disc Cupping (LODC) were the most common abnormalities found in the fundus examination, each observed in $43.75 \%$ of cases. We found no correlation between eye manifestations and Chronic Renal Failure (CRF).

Discussion: There was no direct link between renal failure and eye lesions observed. Several factors explain eye signs, including hypertension and diabetes.

Keywords: Chronic Renal Failure; Eye; Visual Acuity

Abbreviations: HR: Hypertensive Retinopathy; LODC: Large Optic Disc Cupping; CRF: Chronic Renal Failure; IOP: Intra Ocular Pressure; RFA: Retinal Fluorescein
Angiography; AVF: Automated Visual Field; OCT: Optical Coherence Tomography; LVA: Loss of Visual Acuity; SPK: Superficial Punctate Keratitis; HBP: High Blood Pressure. 


\section{Introduction}

Chronic renal failure (CRF) is caused by destructive lesions of the renal parenchyma, induced by primary or secondary nephropathies and by general pathologies such as diabetes, hypertension [1]. It evolves spontaneously to the terminal stage where hemodialysis is essential for the survival of the patient. It is responsible for a chronic uremic syndrome with a series of clinical and biological abnormalities including eye manifestations that can help judge the evolution of the disease [2].

Several studies in sub-Saharan Africa have focused on the description of these eye manifestations, in this case that of Sow Ndiaye in Senegal [2]. In Côte d'Ivoire, we did not find any data available on this topic. In addition, the incidence of CRF is rising sharply due to the sedentary lifestyle of the population, the increase in the prevalence of type II diabetes, hypertension, self-medication with the use of prohibited drugs of the parallel market and uncontrolled use of traditional herbal medicines. Furthermore, the lack of hemodialysis in the majority of patients due to a lack of social coverage, could promote these eye lesions. The purpose of this study was to describe eye lesions observed in patients with chronic renal failure who are not undergoing dialysis, in order to ensure adequate management and thus contribute to improving the quality of life of patients.

\section{Material and Methods}

\section{Material}

Our study was carried out jointly in the Nephrology and Ophthalmology departments of Yopougon University Hospital. It focused on patients with renal failure who are not on dialysis. Were included in the study all patients, regardless of the stage of CRF, regularly followed in the Nephrology department and who underwent a complete ophthalmological examination. Patients with a medical record of nephrology that cannot be used, or who refused to participate in the study, and patients who could not travel to the ophthalmological consultation department because of their bedridden status were not included. Our data collection gathered 60 patients with respect to the selection criteria mentioned above.

\section{Methods}

This was a cross-sectional and descriptive study, which was carried out from February 2017 to June 2017. The data were collected using a survey sheet filled in by a team trained in these departments.
At the ophthalmology department, data collection was done from the clinical examination. Anamnesis revealed socio-demographic features (age, gender and occupation), histories (hypertension, diabetes, HIV/AIDS and others) and functional signs (pruritus, loss of visual acuity). The ophthalmological physical examination was bilateral. It consisted in measuring distance visual acuity with or without correction at the Monnoyer scale. Any subject whose distant visual acuity with correction was less than $3 / 10$ th was considered as a visually impaired subject. Eye motility was appreciated in the different positions of the gaze. Slit-lamp examination made it possible to examine the eyelids and the conjunctiva as well as the anterior segments. Intra ocular pressure (IOP) was measured using Goldman applanation tonometer. Fundus examination was performed in biomicroscopy after pupillary dilatation.

Retinophotography, retinal fluorescein angiography (RFA), automated visual field (AVF), optical coherence tomography (OCT) and corneal pachymetry were the required paraclinical examinations for ophthalmological assessment. We used Kirkendall classification to classify hypertensive retinopathy and arteriosclerosis lesions.

Nephrology data focused on the presumed etiology of chronic renal failure, stage of CRF, serum creatinine, hemoglobin ( $\mathrm{g} / \mathrm{dl})$, serum calcium, uremia, and serum phosphate. Anyone whose hemoglobin level was below $12 \mathrm{~g} / \mathrm{dl}$ was considered anemic.

Data were analyzed with the Epi-Info software version 3.5. Chi-2 and Fisher tests were used to compare quantitative and qualitative variables. The threshold of significance is $5 \%$. Limitations of the study were represented by some patients lost to follow up, the absence of ophthalmological examination of certain patients whose general condition did not allow the transfer to ophthalmological consultation, and incomplete medical records.

\section{Results}

\section{Epidemiological Aspects}

What was the prevalence of ophthalmological involvements in patients?

The male gender was predominant (53\%) with a sex ratio of 1.14. The mean age of patients was $51 \pm 13$ years with age extremes ranging from 17 to 82 years. Workers and craftsmen accounted for the majority of our sample $(40 \%)$, followed by the unemployed $(31.6 \%)$. 


\section{Clinical Aspects}

In our study $88 \%$ of patients were hypertensive and $27 \%$ of patients were diabetic. High blood pressure (hypertension) and diabetes were the main causes of CRF with respective rates of $60 \%$ and $16.6 \%$. Glomerulonephritis and HIV accounted for $13.33 \%$ and $3.3 \%$, respectively of the causes of CRF. In our sample, the majority of patients were at stage 5 of CRF (46.67\%).

We observed that $66.66 \%$ of patients complained of loss of visual acuity (LVA) and $26 \%$ of pruritus. Other functional signs were tearing (15\%), redness $(8.33 \%)$ and eye pain $(6.66 \%)$.

The Slit-lamp examination showed $3.33 \%$ of cases of pterygiun, $1.7 \%$ of superficial punctate keratitis (SPK). On examination of the lens, we observed unilateral cataract in $26.67 \%$ of patients with $56.25 \%$ of partial cataract and $43.75 \%$ of total cataract. The examination of the eyelids, anterior sclera, anterior chamber, pupils, and iris was normal. Eye hypertonia was elevated in $5.83 \%$ of patients. At the posterior pole, optic neuropathies were predominant. It is essentially large Optic Disc Cupping (LODC) (22.95\%) (Table 1).

\begin{tabular}{|c|c|c|c|}
\hline Posterior Pole & Lesions & Numbers & Percentage (\%) \\
\hline \multirow{3}{*}{ Papilla } & LODC & 14 & 23.33 \\
\cline { 2 - 4 } & Pallor & 6 & 10 \\
\cline { 2 - 4 } & Edema & 3 & 5 \\
\hline \multirow{3}{*}{ Macula } & Dry exudates & 6 & 10 \\
\cline { 2 - 4 } & dull & 3 & 5 \\
\cline { 2 - 4 } & scars & 3 & 5 \\
\cline { 2 - 4 } & Hole & 1 & 1.66 \\
\hline
\end{tabular}

Table 1: Distribution of patients according to the posterior pole involvement $(n=60)$.

\begin{tabular}{|c|c|c|c|}
\hline $\begin{array}{c}\text { Peripheral } \\
\text { Chorioretin }\end{array}$ & Lesions & Numbers & $\begin{array}{c}\text { Percentage } \\
\text { (\%) }\end{array}$ \\
\hline \multirow{4}{*}{ Vessels } & Arterial narrowing & 10 & 16.66 \\
\cline { 2 - 4 } & $\begin{array}{c}\text { arteriovenous } \\
\text { Crossing } \\
\text { pathological }\end{array}$ & 2 & 3.33 \\
\cline { 2 - 4 } & Tortuosity & 2 & 3.33 \\
\hline \multirow{4}{*}{ Chorioretinitis } & Exsudates & 5 & 8.33 \\
\cline { 2 - 4 } & Hemorrhage & 5 & 8.33 \\
\cline { 2 - 4 } & Cotton-wool spots & 5 & 8.33 \\
\cline { 2 - 4 } & Pallor & 3 & 5 \\
\cline { 2 - 4 } & Choroidosis & 3 & 5 \\
\hline
\end{tabular}

Table 2: Distribution of patients according to the involvement of the peripheral chorioretin $(n=60)$.
Arterial narrowing (16.66\%) was the most observed lesion in the peripheral chorioretin (Table 2).

\begin{tabular}{|c|c|c|}
\hline Fundus Pathologies & Numbers & Percentage (\%) \\
\hline yypertensive retinopathy & 14 & 43.75 \\
\hline Pathologic LODC & 14 & 43.75 \\
\hline Diabetic retinopathy & 2 & 6.25 \\
\hline Retinal arteriosclerosis & 2 & 6.25 \\
\hline Total & 32 & 100 \\
\hline
\end{tabular}

Table 3: Distribution of patients according to the pathologies of the fundus $(n=32)$.

Hypertensive retinopathy (HR) and large optic disc cupping (LODC) was the most common eye pathologies found on examination of the fundus $(53.84 \%)$.

\begin{tabular}{|c|c|c|}
\hline Eye manifestations & Chi-2 & Probability (p) \\
\hline Loss of visual acuity & 3.169 & 0.366 \\
\hline Pruritis & 3.059 & 0.383 \\
\hline tearing & 2.71 & 0.439 \\
\hline Pterygium & 3.323 & 0.767 \\
\hline Superficial punctate keratitis & 1.169 & 0.762 \\
\hline Cataract & 5.32 & 0.15 \\
\hline Optic neuropathies & 11 & 0.75 \\
\hline Maculopathies & 10.324 & 0.921 \\
\hline Peripheral Chorioretin & 10.167 & 0.601 \\
\hline
\end{tabular}

Table 4: Correlation between eye manifestations and chronic renal failure.

We found no correlation between eye manifestations and CRF stage.

\section{Discussion}

Eye manifestations observed during CRF were polymorphic. In our series, loss of visual acuity (LVA) was the most predominant $(66.66 \%)$ of functional manifestations. This result was higher than that of Sow Ndiaye who noted $44.6 \%$ of cases of LVA [2]. This LVA could be explained on the one hand, by refractive errors found most often in patients with CRF and on the other hand, by the occurrence of certain pathologies such as cataracts. Thus, according to Flament, refractive errors are very common $(30 \%)$ and they would be responsible for a large part of the LVA of patients with renal failure [3]. These refractive errors could be explained by the predominance of the advanced age of our patients. However, according to Flament, pruritus was the dominant functional sign [3]. In our study, it was $26 \%$. 


\section{Open Access Journal of Ophthalmology}

This pruritus is due to an increase in uremia. Mesaric mentioned the responsibility of phosphocalcic disturbances [4]. For him, when the limits of solubility of the phosphocalcic product are exceeded, ubiquitous metastatic calcifications occur. When these calcifications were seated in the skin, the patient complained of skin pruritus. In the eyes, he complained of eye pruritus and Conjunctival hyperemia. LVA $(\mathrm{p}=0.366)$ and pruritus $(\mathrm{p}=$ 0.383) were not significantly related to CRF.

Biomicroscopic examination of the eyelids, conjunctivae and anterior segments of the eyeball revealed cases of pterygium in $3.4 \%$ of the population and a case of superficial punctate keratitis (1.7\%). We therefore note weak Conjunctival manifestations in our series, unlike Flament who reported conjunctivo-corneal calcium deposits in $50 \%$ of patients [3]. These calcium deposits are linked to phosphocalcic metabolic disorders observed during CRF. These deposits may be asymptomatic. But in many cases, they are irritating to the corneal Conjunctival epithelium and would be the cause of diffuse conjunctivitis or localized episcleritis. The corneal Conjunctival lesions vary according to the authors. Ndiaye found Conjunctival calcifications in $15.8 \%$ of the eyes [3]. He also reported other lesions of the conjunctiva including Conjunctival hyperemia in $4.5 \%$, pterygoids in $18.8 \%$ of cases, and pterygium in $2.7 \%$ of cases.

These lesions could be justified by the sociodemographic profile of the chosen sample and the solar exposure factor which is incriminated in the appearance of pterygium. A case of spontaneous sub-Conjunctival hemorrhage was observed by these authors. No significant link was found between conjunctivo-corneal lesions and CRF ( $p=0.767)$ in this series.

The examination of the lens revealed the existence of a cataract in $26.7 \%$ of our population. This proportion was superimposable to that of Ndiaye who reported the presence of cataracts in $26.8 \%$ of eyes [2]. Opacification of the lens in patients with chronic renal failure is related to phosphocalcic metabolism disorders, patient's age, and long-term corticosteroid therapy for pre-existing nephropathy, causal disease and oxidative stress [3]. It is a factor in the loss of visual acuity or even blindness in patients with chronic renal failure according to Costagliola [5]. However, in our study, we found no significant relationship between crystalline lesions and CRF $(\mathrm{p}=0.150)$.

In the fundus, the examination of the optic disc revealed the existence of a large optic disc cupping (LODC) pathological in $23.33 \%$ of cases. This result was superior to that of Ndiaye who noted $5.5 \%$ of LODC cases [2]. This LODC raises fears of glaucomatous optic neuropathy that should be confirmed by performing optical coherence tomography (OCT), visual field examination and central corneal pachymetry. OCT will allow the analysis of the head of the optic nerve, the layer of the retinal nerve fiber and contributes to the positive diagnosis of the condition in addition to visual field examination. The large pathological optic disc cupping sign of optic neuropathy could be justified by nyctohemeral changes in blood pressure. Indeed, hypotension, especially nocturnal or after the institution of a hypotensive treatment, could play a major role in the genesis of the pathological optic disc cupping [3].

Papillary pallor, noted in $10 \%$ of our patients, is due to several causes including toxic and ischemic causes due to anemia and to infectious causes according to Haider [6]. In addition to the large optic disc cupping (LODC) and pallor, papillary edema was observed in 5\% of patients. This result was similar to that of Ndiaye [2]. These latter are the sign of stage III of hypertensive retinopathy according to the Kirkendall classification. They are observed most often at the onset of the disease at the time when the high blood pressure (HBP) is not controlled as was the case in most patients. Indeed, the lack of social care meant that patients were not regularly followed. However, in our study, there was no significant relationship between $\mathrm{CRF}$ and the observed papillary lesions $(p=0.750)$.

At the macular level, we noted $10 \%$ of dry exudates. These could occur at the same period as an existing edema or be exudates of resorption of edema. Dull macula is due to anemia that causes diffuse pallor of the fundus according to Niuta and Flament [7.3]. Macular lesions observed also did not have a significant link with the CRF $(p=0.921)$. These lesions are also described in the literature $[2,3]$.

In the periphery of chorioretin, the most common lesions were retinal hemorrhage, cotton wool spots and dry exudates with proportions of $8.33 \%$ each. They could be the sign of stage 2 of hypertensive retinopathy, according to the Kirkendall classification.

Retinopathies were the most common cases of fundus involvement. Thus, hypertensive retinopathy (HR) accounted for $43.75 \%$ of pathologies of the fundus. As for diabetic retinopathy it was $6.55 \%$. A high frequency of HR has been reported in the literature [3]. Ndiaye also shared this idea with a higher frequency (75.9\%). This predominance of $\mathrm{HR}$ would be due to the presence of 


\section{Open Access Journal of Ophthalmology}

hypertension in most of our patients (88\% of patients' medical history). These lesions are therefore not specific for CRF because the patients were hypertensive and/or diabetic. These are commonly found in vascular and retinal retinopathies including hypertensive retinopathy (stage 2 of the Kirkendall classification) and non proliferative diabetic retinopathy. Moreover, there was no significant relationship between CRF and chorioretinal lesions ( $p=0.601)$. Peripheral retinal pallor was observed in $5 \%$ of patients.

It is one of the most common manifestations of the posterior segment [3]. Ndiaye found $2.67 \%$ of retinal pallor [2]. According to Flament, anemia was the main cause [3].

Retinitis or albuminuric retinopathy is an Ophthalmoscopic image that reflects the impact on the retina of toxemia that results from functional inadequacy of the renal parenchyma. It is thought to be due to azotemia and hypercholesterolemia [8]. In addition, it would be dependent on vascular changes due to hypertension and would result from a nutrition disorder of the retina [8]. The first Ophthalmoscopic evidence of this retinitis was the edema of peri-papillary retinitis, which progressively covers the macular region and gradually spreads to the entire retina. These were hemorrhage and cotton wool exudates that could produce in the macular region the macular star not objectified in this series. The outcome could be favorable with the disappearance of hemorrhage and exudates. All these signs seen in the fundus could be part of albuminuric retinitis in this study.

Still with regard to fundus pathologies, retinal arteriosclerosis was observed in $6.25 \%$ of cases. It was essentially a pathological arteriovenous crossing corresponding to stage 1 of arteriosclerosis according to the Kirkendall classification. Ndiaye found $14.3 \%$ of arteriosclerosis [2]. According to London, the uremic state is responsible for accelerated atherosclerosis, that is, the abnormally early onset compared to the expected effects of age in chronic uremic patients [9]. It would also be promoted by hypertension, diabetes and age [10].

These Arteriovenous crossings could be complicated by retinal vascular occlusions not identified in this study. However, Ndiaye reported the occlusion of the branch of the central artery of the retina in $1.78 \%$ of cases [2]. According to Flament, chronic hypotension and frequent atheroma in patients with chronic renal failure may lead to thrombosis of the central artery of the retina [3]. Other fundus involvements not found in our work have been described particularly optic atrophy (6.4\%), chorioretinal atrophy $(1.78 \%)$, and exudative retinal detachment in one eye. This detachment during the CRF was bilateral and regressed within a few days after the control of hypertension $[2,11]$.

Cases of myopic choroidosis, chorioretinal atrophy, tiger-like appearance of the retina, and macular drusen would be much rarer and without specificity according to Flament [3]. We found $5 \%$ of cases of choroidosis. Cases of anterior ischemic optic neuropathy and ophthalmoplegia have been described by Flament [3]. These optic neuropathies were secondary to hypotension induced by some drugs of hypertension, anemia, and atherosclerosis $[6,12]$. Like Sow Ndiaye, we did not found ophthalmoplegia. However, Flament reported cases of ophthalmoplegia [2,3]. Can also noted a case of bilateral ophthalmoplegia associated with ptosis in a uremic patient [13]. The disappearance of this ophthalmoplegia following dialysis allowed her to also evoke the role of certain toxins in neuropathies of CRF. These toxins were mainly urea, creatinine, parathormone, myosinotol, transketolase and guanidine derivatives. It should be noted that most chorioretinal and papillary changes are not a particular clinical entity and result from hypertension, arteriosclerosis, anemia or even diabetes [3].

With regard to the limitations of this study, it should be noted that some patients in the nephrology department could not benefit from ophthalmological consultation during the study period. This was due to their bedridden state. Examinations such as retinal fluorescein angiography (RFA), OCT and visual field could not be performed. RFA could better help to objectify the retinopathies and optic neuropathies observed in the fundus. OCT could also bring out a macular and / or papillary edema. It would also be necessary with the visual field to diagnose glaucomatous optic neuropathies. This lack of paraclinical examination is explained by the lack of financial means observed in most patients but especially by the absence of a functional exploration block in the ophthalmology department.

\section{Conclusion}

Chronic renal failure is more and more common in our countries. It has multiple consequences on the body especially on the eye. These eye manifestations are rarely mentioned in the general descriptions of CRF. They are sometimes serious, invalidating and irreversible and are mainly represented by hypertensive retinopathy, hence 
the need to perform a perfect blood pressure control in patients with chronic renal failure and to repeat fundus examination regularly.

Other signs have been observed in the fundus, such as papillary pallor, dull macula and peripheral retinal pallor, which are due to anemia which itself is a consequence of CRF. However, these eye manifestations found are not directly related to chronic renal failure. A better follow-up of patients would therefore be necessary to prevent patients from having a double handicap and being a social burden.

\section{References}

1. Maurizi-Balzan J, Zaoui P (2003) Insuffisance rénale chronique, Néphrologie, Corpus médical - Faculté de médecine de Grénoble. Ellipses Ed, 253: 1-6.

2. Sow Ndiaye MN, AM Wane, AM Ka, M Dieng, PA NDoye Roth et al. (2010) Les lésions oculaires chez le patient mélanoderme atteint d'insuffisance rénale chronique. Mali Med 25(4): 14-20.

3. Flament J, Storck D (1997) CEil et pathologie générale. Rapport SFO, Masson, Paris, pp: 494-417.

4. Mesaric B (1974) Examen systématique des modifications pathologiques des yeux des malades souffrant d'insuffisance rénale chronique. Arch Ophthalmol 12: 907-916.
5. Costagliola C, Juliano G, Menzione M, Simonelli F, Tortori A, et al. (1990) Systemic human diseases as oxidative risk factors in catractogenesis. II. Chronic renal failure. Exp Eye Res 51(6): 631-635.

6. Haider S, Astbury NJ, Hamilton DV (1993) Optic neuropathy in uraemic patients on dialysis. Eye $7(1)$ : 148-151.

7. Niutta A, Spicci D, Barcarolli I (1993) Fluorographic findings in hemodialyzed patients. Ann Ophthalmol 25: 375-380.

8. Pathogénie de la rétinite albuminurique.

9. London GM, Marchais SJ, Safar ME, Genest AF, Guerin AP, et al (1990) Aortic and large artery compliance in end-stage renal failure. Kidney Int 37(1): 137-142.

10. Ross $R$ (1986) The pathogenesis of atherosclerosis-an update. N Engl J Med 314(8): 488-500.

11. Regenbogen L, Coscas G, Debbasch S (1995) OEil et rein. Encycl. Med Chir (Paris - France), Ophtalmologie, 21-453-A-25; 9.

12. Vrabec R, Vatavuk Z, Pavlovic D, Sesar A, Cala S, et al. (2005) Ocula findings in patients with chronic renal failure undergoing haemodialysis. Coll Antropol 29(1): 95-98.

13. Can U, Aydin P, Kansu T (1994) Bilateral external ophthalmoplegia in uremia. Nephron 68(3): 391. 